\title{
Performance Evaluation of a Small Scale Modular Solar Trigeneration System
}

\author{
Handong Wang \\ M. \& E. School of Shenzhen Polytechnic, Shenzhen, Guangdong 518055, China \\ Correspondence should be addressed to Handong Wang; zlwhd@szpt.edu.cn
}

Received 5 April 2014; Accepted 7 May 2014; Published 25 May 2014

Academic Editor: Mohammad Yusri Hassan

Copyright (c) 2014 Handong Wang. This is an open access article distributed under the Creative Commons Attribution License, which permits unrestricted use, distribution, and reproduction in any medium, provided the original work is properly cited.

\begin{abstract}
In order to improve the efficiency of solar thermal power (STP) system, a novel modular system combining cooling, heating, and power generation (CCHP) is proposed and introduced in this work. This modular CCHP system can simultaneously provide $10 \mathrm{~kW}$ electricity, $-15 \sim 5^{\circ} \mathrm{C}$ coolant, and $60^{\circ} \mathrm{C}$ hot water to meet the requirements of cooling, heating, and electricity in a general family or other fields. The flow chart and working process of the modular system are introduced, based on which the energy and exergy efficiencies at the CCHP and STP operation modes are primarily evaluated and discussed. The results show that when the output electricity is constant, the overall efficiencies of energy and exergy of the system operating at the CCHP mode are 9.37 times and 2.62 times as big as those of the system operating at the STP mode, respectively. Thus, the modular solar thermal CCHP system can improve the energy and exergy efficiencies. Furthermore, calculation shows that both the overall energy and exergy efficiencies decrease with increase of inlet vapor temperature at given inlet vapor pressure, but both the efficiencies increase with increase of inlet vapor pressure at given inlet temperature.
\end{abstract}

\section{Introduction}

Solar thermal power (STP) systems produce electricity (power) in much the same way as conventional power stations. The difference is that STP systems obtain energy by concentrating solar radiation and converting it to high temperature steam or gas to drive a turbine or engine. In STP systems, the incoming radiation is tracked by mirror fields which concentrate the energy towards collectors or absorbers. Then, the received solar radiation is transferred thermally to the working medium. The heated fluid operates as in conventional power stations directly (if steam or air is used as a medium) or indirectly through a heat exchanging steam generator on the turbine unit which then drives the generator (i.e., a turbine or engine). In STP systems, the collected and transferred thermal energy can be stored with special thermal tanks which can continuously supply high temperature working medium to drive the generator for several hours after sunset. Compared to photovoltaic (PV) power systems, the characteristics of electricity generated by STP systems are almost the same as those of electricity generated by traditional power stations. Thus, the output electricity can be supplied to a power net without inverters which are necessary in PV power systems. As it can avoid environment pollution problems caused by fossil fuel power systems, the STP technology is considered as one of the greenest technologies in power fields.

There are many researches about STP systems. Among them, Pons and Clark proposed a system based on dish solar collectors and Stirling engines [1]. Goswami presented a study on solar thermal power. In his work, a relatively recent cycle has been proposed in which thermal energy is used to produce work and to generate a subambient temperature stream that is suitable for cooling applications [2]. You and Hu studied a medium-temperature solar thermal power system and its efficiency optimization; they declared that the optimum saturation temperature in the boiler was about $201^{\circ} \mathrm{C}$ and the thermal efficiency and the exergetic efficiency of the system were $17.9 \%$ and $25.12 \%$, respectively [3].

$\mathrm{Lu}$ and Goswami investigated the optimization of a novel combined power-refrigeration thermodynamic cycle and cooling thermodynamic cycle for low temperature heat sources [4]. Tamm et al. published the theoretical and 
experimental results of a novel combined power and cooling thermodynamic cycle for low temperature heat sources in $[5,6]$, respectively. Vijayaraghavan and Goswami presented a research on organic working fluids for a combined power and cooling cycle and found that, under optimum conditions, thermodynamic efficiencies achievable with organic fluid mixtures were lower than those obtained with ammoniawater mixtures, but the refrigeration temperatures achievable using organic fluid mixtures were higher than those using ammonia-water mixtures [7]. Kane et al. also presented a small hybrid solar power system [8].

There are also many researches on STP systems based on ammonia-water power cycle. For example, Xu and Goswami investigated the thermodynamic property of ammonia-water mixtures for power cycle applications [9]. Analysis of a new thermodynamic cycle for combined power and cooling using low and mid temperature solar collectors is studied by Goswami and Xu [10] and by Xu et al. [11]. Ammonia-based combined power-refrigeration cycle at low refrigeration temperatures is analyzed by $\mathrm{Lu}$ and Goswami [12]. A novel ammonia-water cycle for power and refrigeration cogeneration is presented by Zhang et al. [13]. Abdel-Rehim also investigated an ammonia-water-based power-refrigeration system with parabolic trough solar collectors [14].

In recent years, Mayere and Riffat proposed a microcombined heating-power system with output capacity less than $10 \mathrm{~kW}$ for domestic power generation. They declared that the systems could utilize about $75 \%$ of solar energy to provide electric and thermal energy directly to end-users, and the microsystems had relatively the same efficiencies compared to large scale systems which were said to be matured technologies [15]. Borello et al. investigated the matching of an advanced small scale combined heat and power (CHP) Rankine cycle plant with end-user (i.e., a hotel) thermal and electric load [16].

As we know, most of researches on CCHP systems are mainly about the system with combustion power generator, but few are about solar thermal CCHP systems. Among few references about the solar thermal CCHP system, Su et al. proposed and discussed a distributed energy system for cooling-heating-power cogeneration for a single building [17]. In their work, solar energy is used as the only heat source to drive a closed Brayton cycle to generate power. The waste heat dissipated by the turbine is utilized by a recovery refrigeration cycle to provide cooling or is directly transferred by heat exchangers for heating. When the system is further combined with a fuel cell system, the whole system can provide cooling-heating power continuously in day and night. In fact, the system proposed in [17] is just a coolingpower or heating-power cogeneration system which cannot supply cooling, heating, and power simultaneously.

References review indicates that most of STP plants are large systems which need huge areas to install mirror fields. In order to investigate the feasibility and efficiency of small scale systems, a micromodular solar thermal CCHP system is proposed and introduced in this work. This microsolar thermal CCHP system can meet the requirements of families, farms, islands, and other places lack of electricity. In this work, the flow chart and working process of the modular solar thermal CCHP system are firstly introduced. And, then, the performances at typical conditions are primarily evaluated and discussed.

\section{Description and Working Process of the Modular CCHP System}

In order to meet the demands of power, cooling, and heating in general families or small farms, the function of the modular solar thermal CCHP system is given as follows: supplying $10 \mathrm{~kW}$ electricity, $-15 \sim 5^{\circ} \mathrm{C}$ coolant, and $60^{\circ} \mathrm{C}$ hot water. This part mainly presents the conceptual design and working process of the modular solar thermal CCHP system. At present, each module is designed to regularly generate $10 \mathrm{~kW}$ electricity. It mainly consists of solar collectors and absorbers, thermal energy storage tanks, a vapor generator (heat exchanger or boiler), a power generator, an absorption refrigerator, a water heater, and a cooling unit, as shown in Figure 1. In this CCHP system, the power generation is based on the principle of Rankine cycle. This is different from the system presented in [17] which was based on the closed Brayton cycle.

The modular CCHP system can be divided into two parts. The first part is called solar thermal unit (STU) which includes the solar collectors, thermal storage tanks, heat exchanger (vapor generator or boiler), and the first fluid pump. The second part is called power and end-users (PEU) which consists of the heat exchanger, power generator, DAR (diffusion absorption refrigerator), water heater, cooling unit, and the second fluid pump. In the first part (STU), the first fluid flows through the solar collectors and absorbers and becomes high-temperature fluid which releases heat to the second fluid in the heat exchanger and is subsequently pumped to the solar collector to begin the next circulation. When solar energy is sufficient, the first fluid can be pumped into the thermal energy storage tanks and the excess thermal energy can be stored for continuously generating power, cooling, and heating after sunset.

In the second part (PEU), the second fluid becomes vapor (or steam) after being heated by the first fluid in the heat exchanger. Then, the vapor drives the power generator to produce electricity. The exhausted vapor from the power generator subsequently enters into the absorption refrigerator to produce cooling and supply low temperature coolant to users. The second fluid leaving from the absorption refrigerator may still be a mixture of vapor and hot water, and its temperature is still high enough to produce hot water in the water heater by exchanging heat with the external circulating water. If necessary, the second fluid leaving the water heater enters into the cooling unit to be condensed thoroughly. Finally, the second fluid liquid is pumped back to the heat exchanger (vapor generator) to begin the next circulation. Of course, if the second fluid completely becomes hot water when it leaves the water heater, the cooling unit can be shut off to save energy. This is also one of the advantages of the modular solar thermal CCHP system. Thus, the first part and the second part can be integrated into one system by the heat exchanger (i.e., vapor generator or boiler). 


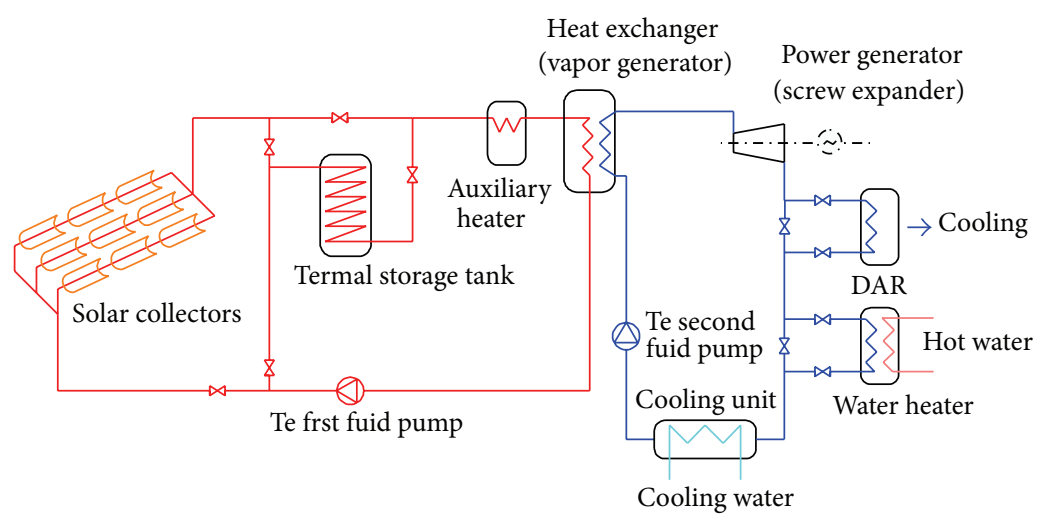

FIGURE 1: Conceptual flow chart of the modular solar thermal CCHP system.

At present, parabolic trough or dish reflectors with heat absorbers are chosen to be the solar collectors and absorbers in which thermal oil is chosen as the first fluid. The designed temperature of the first fluid is $150 \sim 250^{\circ} \mathrm{C}$. The second fluid can be water or other organic substances with low-boiling temperatures based on the available temperature of the first fluid. The heat exchanger is used to generate vapor and it can be a shell-tube or a plate-type heat exchanger. The thermal storage tanks are used to store excess thermal energy for generating power after sunset. One kind of phase-change materials is chosen as the thermal-store medium in the thermal storage tanks. It can reduce the occupied area of the tanks. An auxiliary heater is equipped to reheat the first fluid if necessary. The auxiliary heater can be a natural gas or an oil driven heater.

The power generator is the key equipment of the system. It is difficult to find a suitable microvapor turbine as the output electricity is less than $10 \mathrm{~kW}$ in each CCHP module. But fortunately, we have noticed that there is a manufacturer who can provide one kind of microscrew engine with output power of $10 \mathrm{~kW}$. Furthermore, the driving medium of screw engines can be vapor or mixture of vapor and water. Thus, a $10 \mathrm{~kW}$ microscrew engine is chosen to be the power generator in the modular solar thermal CCHP system.

Water heater is mainly a heat exchanger used to heat the external circulating water. A plate type heat exchanger can be used as water heater in the modular CCHP system.

Of course, pumps are necessary to drive the fluids in the modular solar thermal CCHP system. The main pumps include a first fluid pump, a second fluid pump, a coolant pump and solution pumps in the absorption refrigerator, a hot water pump in the water heater, and a cooling water pump in the cooling unit.

If the refrigerator should meet the requirements of freezing/chilling food and air conditioning, the temperature of coolant should be lower than $0^{\circ} \mathrm{C}$. Thus, general $\mathrm{LiBr}-\mathrm{H}_{2} \mathrm{O}$ absorption refrigerators with the evaporating temperature above $0^{\circ} \mathrm{C}$ cannot be used at this field though its efficiency is relatively higher. A novel diffusion-absorption refrigerator (DAR) proposed by the author is used to supply low temperature coolant in the modular solar thermal CCHP system. The diagram of flow chart of the novel DAR is shown in
Figure 2. Experimental researches have shown that this novel DAR can be driven by $72 \sim 96^{\circ} \mathrm{C}$ hot water to provide $-13 \sim 5^{\circ} \mathrm{C}$ coolant [18]. Because the temperature of the second fluid leaving from the power generator is higher than $96^{\circ} \mathrm{C},-15 \sim$ $5^{\circ} \mathrm{C}$ coolant is feasibly obtained in the modular CCHP system. Furthermore, as the DAR has unitary pressure in the whole system, the pumps in the novel DAR are just used to overcome the flowing resistance of the fluids. Thus, the pumps in the novel DAR consume less electricity than the pumps used in other absorption refrigerators.

The novel DAR consists of a generator, a condenser, an adiabatic spray evaporator and an evaporator heat exchanger, an adiabatic spray absorber and a solution cooler, a solution heat exchanger, a reservoir, and circulation pumps, in which the generator, condenser, evaporator heat exchanger, solution cooler, and solution heat exchanger are plate heat exchangers. The working fluid is the triple fluid of $\mathrm{LiNO}_{3}-\mathrm{NH}_{3}-\mathrm{He}$, in which $\mathrm{LiNO}_{3}, \mathrm{NH}_{3}$, and $\mathrm{He}$ (helium) are used as absorbent, refrigerant, and diffusion gas, respectively. The principle or working process of the DAR shown in Figure 2 can be described as follows.

At first, the hot water or vapor enters into the generator and heats the strong solution (high concentration of ammonia). When the strong solution temperature reaches its saturate point, ammonia vapor escapes and separates from solution. Vapor is condensed by cooling water in the condenser and liquid ammonia subsequently enters the spray evaporator in which diffusion and evaporation take place and some liquid ammonia evaporates to cool down the remaining liquid ammonia. Then the remaining liquid ammonia with low temperature is pumped into the evaporator heat exchanger to exchange heat with the coolant and subsequently goes back to the evaporator to take part in the next diffusion-evaporation process. And the evaporated ammonia vapor in the evaporator mixes with the diffusion gas Helium and then flows into the spray absorber by one of the gas pipes. At the same time, the weak solution separated from the generator flows through the solution heat exchanger and is cooled down by the strong solution pumped out from reservoir. The precooled weak solution is then pumped through the solution cooler and subcooled by cooling water and subsequently sprayed into the spray absorber. In spray 


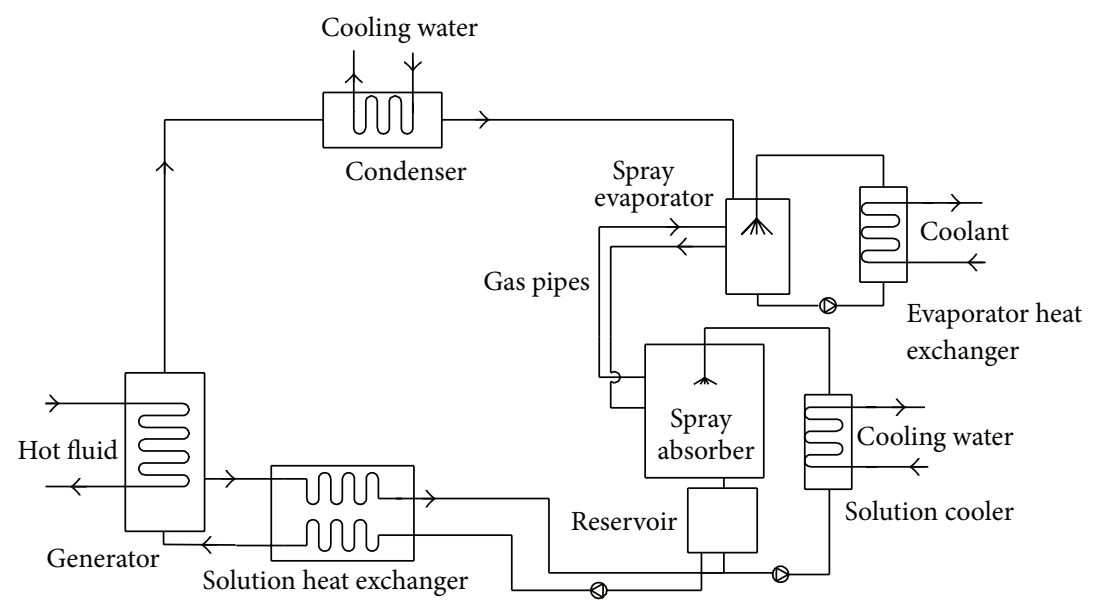

FIGURE 2: Diagram of flow chart of the novel DAR.

absorber, the falling weak solution absorbs ammonia vapor from gas mixture $\left(\mathrm{NH}_{3}\right.$ and $\left.\mathrm{He}\right)$ and becomes strong solution at the outlet of absorber, while the gas mixture with less ammonia, because of its smaller density, rises and enters into the evaporator by the other gas pipe to take part in the next diffusion-absorption process. The strong solution then leaves absorber and enters into the reservoir and is consequently pumped into the generator after getting preheated by the high temperature weak solution in the solution heat exchanger. It can be seen that there are two circulations in the operation. One is the solution circulation in the circuit consisting of the generator, vapor-solution separator, solution heat exchanger, solution cooler, spray absorber, and reservoir. The other is the gas circulation in the circuit mainly consisting of the spray absorber and evaporator.

DAR has advantages such as single-pressure, having no need of throttling valve, little noise, easy maintenance, and long service life. The equipped low power consumption pumps not only increase the amount of solution circulation and make it possible to enlarge refrigeration capacity, but also avoid the strict limitation of relative position of each part. Moreover, the use of an adiabatic spray absorber and plate heat exchangers makes it more compact. It should be mentioned that the circulation pumps in this DAR are just used to overcome the flow resistance. This is different to the ones used in other absorption refrigeration systems in which the pumps should provide higher pressure to build high pressure region. So the input power of each pump in Figure 2 is very small, which can obviously reduce electricity consumption.

The operation modes of the modular CCHP system shown in Figure 1 are very flexible and it can be easily changed from one to another as follows:

(a) combined cooling, heating, and power generation (CCHP);

(b) combined cooling and power generation (CCP);

(c) combined heating and power generation (CHP);

(d) combined cooling and heating $(\mathrm{CCH})$; (e) power generation (this mode is equivalent to a general STP system);

(f) cooling;

(g) heating.

In each operation mode, the thermal energy storage can be determined to operate or shut off according to the collected solar energy. This modular system can simultaneously provide $10 \mathrm{~kW}$ electricity, $-15 \sim 5^{\circ} \mathrm{C}$ coolant, and $60^{\circ} \mathrm{C}$ hot water to meet the requirements of cooling, heating, and power consumption in a general family or other fields. It also can be assembled to be a bigger system by connecting several modules together to meet the requirements of different fields.

Based on this work, we are designing and constructing a prototype of this modular trigeneration system in our campus. Further theoretical and experimental researches will be conducted in the future.

\section{Performance Evaluation and Discussion}

As mentioned above, the second fluid in Figure 1 may be water or an organic working fluid, but water $\left(\mathrm{H}_{2} \mathrm{O}\right)$ was chosen as the second fluid (i.e., the power cycle medium) to evaluate the performance of the solar thermal CCHP system. The reason is that this is a trigeneration system, and the DAR should be driven by heat source with temperature higher than $96^{\circ} \mathrm{C}$ to obtain $-15^{\circ} \mathrm{C}$ coolant. If an organic working fluid is chosen as the second fluid, the temperature of the second fluid at outlet of the power generator will be too low to drive the DAR to obtain $-15^{\circ} \mathrm{C}$ coolant. For example, if the second fluid is $\mathrm{R} 245 \mathrm{fa}$ and its temperature at inlet of the power generator is $150^{\circ} \mathrm{C}$, the temperature at outlet of the power generator is just about $83^{\circ} \mathrm{C}$ when the expansion radio of the power generator is given as 4 . Though this outlet temperature $\left(83^{\circ} \mathrm{C}\right)$ can start the DAR, it is not high enough to drive the DAR to obtain $-15^{\circ} \mathrm{C}$ coolant. Thus, water was chosen as the second fluid to meet the trigeneration requirement. In other words, if the required coolant temperature is above $0^{\circ} \mathrm{C}$, an organic working fluid such as R245fa may be an ideal candidate of the second fluid. 
TABLE 1: Parameters of the second fluid at the inlet/outlet of each part.

\begin{tabular}{|c|c|c|c|}
\hline State point & Pressure $(\mathrm{MPa})$ & Temperature $\left({ }^{\circ} \mathrm{C}\right)$ & Medium and dryness \\
\hline 1 & 0.6 & 160 & Overheated vapor, $x^{*}=1$ \\
\hline 2 & 0.15 & 111.35 & Wet vapor, $x=0.9482$ \\
\hline $2 \mathrm{a}$ & 0.15 & 111.35 & Wet vapor, $x=0.5$ \\
\hline 4 & 0.15 & 111.35 & Saturated water, $x=0$ \\
\hline 5 & 0.6 & 111.42 & Subcooled water, $x=0$ \\
\hline 6 & 0.6 & 158.83 & Saturated water, $x=0$ \\
\hline
\end{tabular}

TABLE 2: Input power of each pump.

\begin{tabular}{lcccccc}
\hline & \multicolumn{2}{c}{ Pumps in DAR } & Cooling water \\
pump & Absorber & $\begin{array}{c}\text { Solution } \\
\text { pump }\end{array}$ & $\begin{array}{c}\text { Evaporator } \\
\text { pump }\end{array}$ & $\begin{array}{c}\text { Hot } \\
\text { water pump }\end{array}$ & $\begin{array}{c}\text { The second } \\
\text { fluid pump }\end{array}$ & $\begin{array}{c}\text { The first } \\
\text { fluid pump }\end{array}$ \\
\hline $\begin{array}{l}\text { Input power } \\
\text { (kW) }\end{array}$ & 0.37 & 0.09 & 0.09 & 1.1 & 0.09 & 2.2 \\
\hline
\end{tabular}

In addition, attentions were mainly paid to the comparison of CCHP and STP modes. In other words, only the operation modes of A (i.e., CCHP mode) and E (i.e., STP mode) mentioned above are considered in the next evaluation to compare the efficiencies of CCHP and STP systems. It also should be mentioned that the effect of thermal storage and auxiliary heater is not taken into account at this work when evaluating the performance of the solar thermal CCHP system.

3.1. Assumptions and Calculation Methodology. Before evaluating the performance, some assumptions are given as follows.

(1) The total output electricity is given as $10 \mathrm{~kW}$. The expansion ratio, expansion efficiency, and the workto-electric efficiency of the power generator are 4.0, 0.75 , and 1.0 , respectively.

(2) The thermal energy collected by solar collectors is adequate for power generation. The input energy of the system is calculated by the input energy of vapor flowing through the power generator.

(3) The efficiencies of the novel DAR and the heat exchanger in water heater are 0.3 and 0.8 , respectively.

(4) The flow resistance of the second fluid is neglected.

(5) The efficiency of each pump is given as 0.75 .

The power cycle of the second fluid (water) in the temperature-entropy $(T-s)$ diagram is shown in Figure 3. The parameters of the second fluid at the inlet/outlet of each part are given in Table 1 . The rated input power of each pump is given in Table 2.

When the system operates in mode E (i.e., power generation or STP mode), the vapor leaving the power generator is still assumed to be wet vapor. The parameters in this mode are shown in Table 1. The STP cycle consists of the cycle 1-2-4-5-6-7-1 as shown in Figure 3. In the cycle, 2-4 is a cooling process and the cooling unit in Figure 1 should

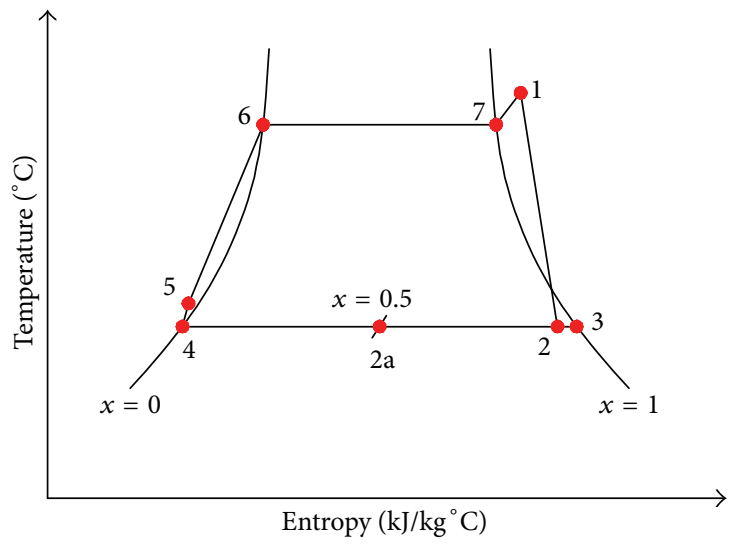

FIgURE 3: Power cycle of the second fluid (water).

operate to condense the exhausted vapor leaving from the power generator in the STP mode. Thus, the condensed saturated water can be reused and circulated in the second part (PEU) of the system.

In Figure 3, the total input thermal energy of the system can be determined by

$$
Q_{v, \text { in }}=m_{s}\left(h_{1}-h_{5}\right) \text {, }
$$

where $Q_{v \text {,in }}$ is the total input thermal energy of the system, $\mathrm{kW} ; m_{s}$ is the mass flow rate of the second fluid (steam or water), $\mathrm{kg} / \mathrm{s}$; and $h_{1}$ and $h_{5}$ are the specific enthalpies of state points of 1 and 5 , respectively, $\mathrm{kJ} /\left(\mathrm{kg}^{\circ} \mathrm{C}\right)$.

The power output of the system $N_{\mathrm{PG}}$ can be calculated by

$$
N_{\mathrm{PG}}=m_{s}\left(h_{1}-h_{2}\right) \text {. }
$$

If the thermal energy of processes of 2-2a and $2 a-4$ in Figure 3 are used to drive the DAR and the water heater, 
respectively, the cooling capacity of the DAR and heating capacity of the water heater can be calculated by, respectively,

$$
\begin{gathered}
Q_{C}=m_{s}\left(h_{2}-h_{2 a}\right) \eta_{\mathrm{DAR}}, \\
Q_{\mathrm{HW}}=m_{s}\left(h_{2 a}-h_{4}\right) \eta_{\mathrm{WH}},
\end{gathered}
$$

where $Q_{C}$ is the cooling capacity of the refrigerator, $\mathrm{kW}$, and $Q_{H W}$ is the heating capacity of the water heater, $\mathrm{kW} . \eta_{\mathrm{DAR}}$ and $\eta_{\mathrm{WH}}$ are the efficiencies of DAR and water heater, respectively.

The overall energy efficiency (i.e., the first law efficiency) of the CCHP system is determined by

$$
\eta_{S}=\frac{N_{\mathrm{PG}}+Q_{C}+Q_{\mathrm{HW}}-\sum N_{p, i}}{Q_{v, \text { in }}},
$$

where $\eta_{s}$ is the overall energy efficiency of the solar thermal CCHP system and $\sum N_{p, i}$ is the total input power of pumps, $\mathrm{kW}$. The input power of the second fluid pump $\left(N_{p, \mathrm{sec}}\right)$ depends on the vapor flow rate and it can be calculated as

$$
N_{p, \text { sec }}=\frac{1000 m_{s}\left(p_{5}-p_{4}\right)}{\rho_{4}},
$$

where $p_{4}$ and $p_{5}$ are the inlet pressure and outlet pressure of the second fluid pump (as shown in Figure 3), $\mathrm{MPa}$, and $\rho_{4}$ is the density of the second fluid at the inlet of the pump, $\mathrm{kg} / \mathrm{m}^{3}$.

Each input power of the other pumps is calculated based on its rated input power as shown in Table 2.

The overall exergy efficiency (i.e., the second law efficiency) of the solar thermal CCHP system is determined by

$$
\eta_{\mathrm{ex}}=\frac{N_{\mathrm{PG}}+E_{\mathrm{C}}+E_{\mathrm{HW}}-\sum N_{p, i}}{E_{v, \text { in }}},
$$

where $\eta_{\mathrm{ex}}$ is the overall exergy efficiency of the solar thermal CCHP system; $E_{C}$ is the exergy of cooling capacity of the refrigerator, $\mathrm{kW} ; E_{\mathrm{HW}}$ is the exergy of heating capacity of the water heater, $\mathrm{kW} ; E_{v \text {,in }}$ is the exergy of input thermal energy of the system, $\mathrm{kW}$; and $\Sigma N_{p, i}$ is the total exergy of input power of pumps, $\mathrm{kW}$.

Thus, the $E_{C}, E_{\mathrm{HW}}$, and $E_{v \text {,in }}$ in (6) can be calculated as, respectively,

$$
\begin{gathered}
E_{C}=Q_{C}\left(\frac{T_{0}+273.15}{T_{e}+273.15}-1\right), \\
E_{\mathrm{HW}}=Q_{\mathrm{HW}}\left(1-\frac{T_{0}+273.15}{T_{\mathrm{HW}}+273.15}\right), \\
E_{v, \text { in }}=Q_{v, \text { in }}\left(1-\frac{T_{0}+273.15}{T_{\mathrm{hs}}+273.15}\right),
\end{gathered}
$$

where $T_{0}, T_{e}, T_{\mathrm{HW}}$, and $T_{\mathrm{hs}}$ are the average temperatures of environment, evaporator, hot water, and hot fluid in the heat exchanger (vapor generator), respectively. In this case study, the $T_{0}, T_{e}$, and $T_{\mathrm{HW}}$ are given as $25^{\circ} \mathrm{C},-10^{\circ} \mathrm{C}$, and $111.35^{\circ} \mathrm{C}$, respectively. The averaged $T_{\mathrm{hs}}$ is given to be $10{ }^{\circ} \mathrm{C}$ higher than the temperature of vapor at outlet of the vapor generator; that is, $T_{\mathrm{hs}}=T_{1}+10$.

\subsection{Results and Discussion}

3.2.1. Performance Comparison of CCHP and STP Modes. Based on the above conditions, primary calculation shows that when the system operates in the mode of $E$ (i.e., equivalent STP mode) and generates $10 \mathrm{~kW}$ electricity, the necessary input vapor energy is $126.42 \mathrm{~kW}$. Under the conditions shown in Tables 1 and 2, the energy and exergy utilization profiles for operation modes $\mathrm{A}$ and $\mathrm{E}$ are shown in Figures 4 and 5, respectively.

The pumps operating in the mode E consume energy of $2.235 \mathrm{~kW}$ so that the net output electricity is $7.765 \mathrm{~kW}$. Thus, the overall energy efficiency of the system $\left(\eta_{s}\right)$ in the mode $\mathrm{E}$ is only $6.14 \%$. Moreover, tremendous thermal energy should be released by cooling water to condense the exhausted vapor of the power gen erator. In this case, the thermal energy released by cooling water is $92.09 \%$ of the input energy. The energy consumption of cooling water pump and the released thermal energy seriously deteriorate the overall energy efficiency of solar energy utilization. In this STP mode, the overall exergy efficiency of the system is $18.77 \%$ which is less than the results of [3]. In [3], the exergy efficiency was $25.12 \%$ but the saturation temperature in the boiler was about $201^{\circ} \mathrm{C}$ which is higher than that in this work (namely, $160^{\circ} \mathrm{C}$ ).

When the system operates in the mode A (CCHP mode) and the working conditions are as shown in Table 1, the cooling unit can be shut off because of the subsequent utilization of refrigerator and water heater. Calculation shows that in the CCHP mode the system can produce $10 \mathrm{~kW}$ electricity, $16.51 \mathrm{~kW}$ cooling, and $49.13 \mathrm{~kW}$ heating, and the overall energy efficiency of the system reaches $57.56 \%$ which is 9.37 times as big as that of mode E (STP mode). In the CCHP mode, the pumps consume $2.875 \mathrm{~kW}$ and the net output electricity is $7.125 \mathrm{~kW}$. Though the net output electricity of the CCHP mode is less than that of the STP mode, the extra recovered cooling and heating promote obviously the overall efficiency of the CCHP mode. The gained heating capacity in water heater can heat city water from $20^{\circ} \mathrm{C}$ to $60^{\circ} \mathrm{C}$ with flow rate of $1.05 \mathrm{~m}^{3} / \mathrm{h}$. In the CCHP mode, the overall exergy efficiency is $49.21 \%$ which is 2.62 times as big as that of the STP mode. It is obvious that the CCHP mode can significantly improve the performance of the solar thermal CCHP system.

Figures 4 and 5 show that when the system operates in mode E (STP mode), the losses of energy and exergy are $92.09 \%$ and $75.82 \%$, respectively. But when it operates in mode A (CCHP mode), the losses of energy and exergy are only $40.17 \%$ and $43.84 \%$, respectively. So the CCHP operation mode can significantly improve the energy utilization efficiency.

In addition, if the coolant temperature is above $0^{\circ} \mathrm{C}$, for example, just meeting the requirement of air conditioning, the DAR can be replaced by a high efficiency $\mathrm{LiBr}-\mathrm{H}_{2} \mathrm{O}$ absorption refrigerator. Thus, the overall efficiency of the system can be promoted greatly. For example, if the coefficient of performance (COP) of $\mathrm{LiBr}-\mathrm{H}_{2} \mathrm{O}$ absorption refrigerator is given as 1.0 with $5^{\circ} \mathrm{C}$ evaporator temperature and the total 


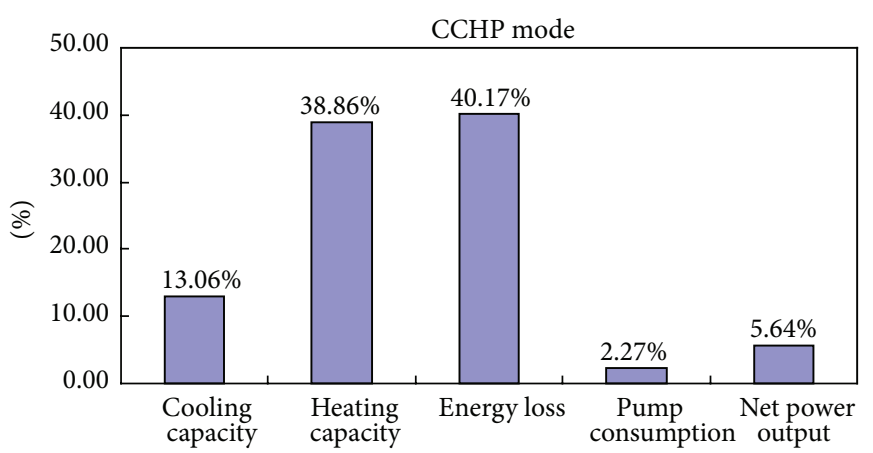

(a)

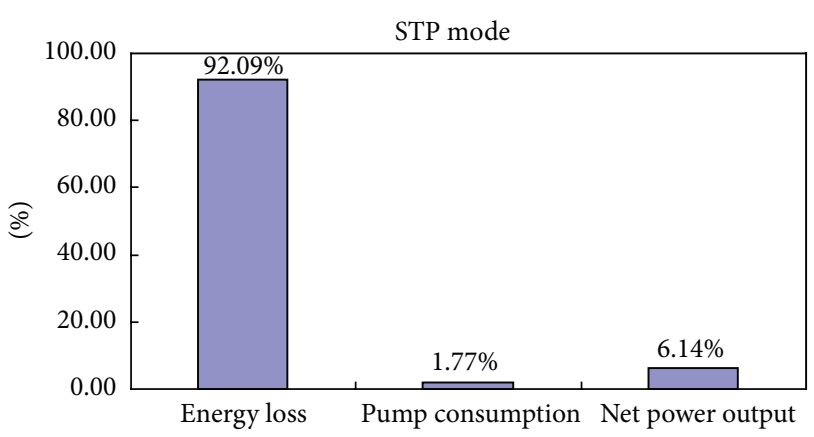

(b)

FIGURE 4: Energy utilization profiles of operation modes A (CCHP) and E (STP).

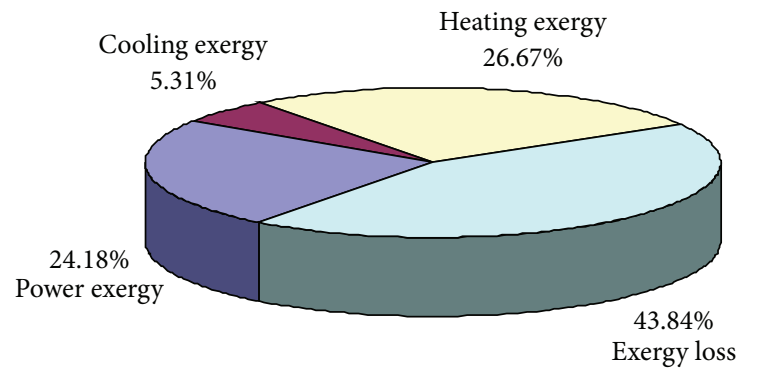

(a) CCHP mode

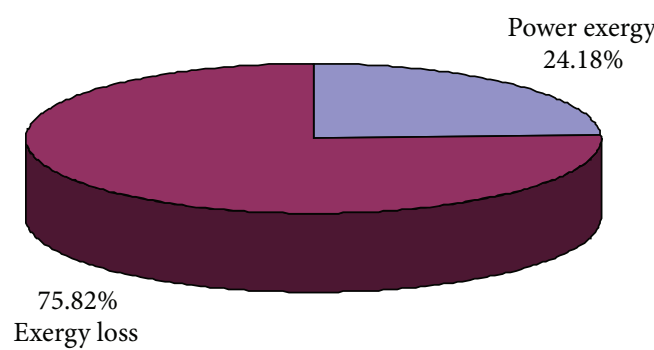

(b) STP mode

FIGURE 5: Exergy utilization profiles of operation modes A and E.

input power of the additional solution pumps is $5 \mathrm{~kW}$, the overall energy efficiency and exergy efficiency of the CCHP system can reach $84.59 \%$ and $53.47 \%$, respectively. It sounds more attractive.

3.2.2. Influence of Inlet Vapor Parameters. Calculations show that when the total output electricity is given as $10 \mathrm{~kW}$ and the expansion ratio of the screw expander (power generator) is 4.0 , the efficiencies of the STP or CCHP mode vary with the inlet pressure and temperature of vapor entering the power generator, as shown in Figure 6.

Figures 6(a) and 6(b) illustrate that when the inlet vapor temperature $\left(T_{\text {in }}\right)$ is constant, all the energy and exergy efficiencies of the CCHP and STP mode increase with inlet vapor pressure increasing. But it is not very obvious for the STP mode or the overall energy efficiency of the CCHP mode. The overall exergy efficiency of CCHP mode increases rabidly with inlet vapor pressure increasing. It means that higher inlet vapor pressure can improve the efficiencies of the CCHP and STP modes, especially the exergy efficiency of the CCHP mode.

Figure 6(c) shows that the exergy efficiency of STP mode decreases obviously with the inlet vapor temperature when the inlet vapor pressure is constant though the energy efficiency of STP mode slightly increases with the inlet vapor temperature increasing. The reason is that the input heat capacity (i.e., input energy) decreases about $11.37 \%$, while the input exergy increases $32.39 \%$ when the inlet temperature varies from $160^{\circ} \mathrm{C}$ to $300^{\circ} \mathrm{C}$. Figure $6(\mathrm{~d})$ shows that the higher the inlet vapor temperature, the smaller the overall energy and exergy efficiencies of CCHP mode if the inlet vapor pressure is constant. Figures $6(\mathrm{c})$ and $6(\mathrm{~d})$ illustrate that the exergy efficiencies of both CCHP and STP modes cannot benefit from a higher inlet vapor temperature at given conditions. Calculations also indicate that it is suitable to keep the inlet vapor temperature the same as the saturate temperature at given inlet vapor pressure. Though this may cause the status of end-expansion to be in the wet vapor region, it is also acceptable because the screw expander can operate with wet vapor without any problem.

Figure 6 indicates that the CCHP system can benefit from higher inlet vapor pressure, but not the higher inlet vapor temperature. It also means that in this solar thermal CCHP system, the temperature of the first fluid in solar collectors should not be much higher than the corresponding saturate temperature of the inlet vapor of the screw expander. The detailed effect and optimum parameters need to be investigated in future theoretical and experimental researches.

\section{Conclusions}

The above analyses indicate that the proposed micromodular solar thermal CCHP system is feasible and it can significantly improve the energy and exergy efficiencies of solar thermal 


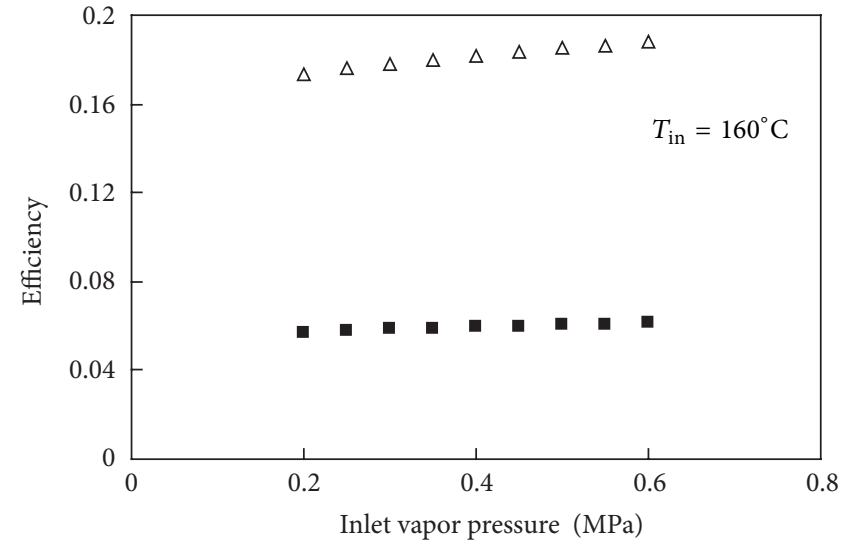

$\triangle \eta_{\text {ex }}$ of STP mode

(a)

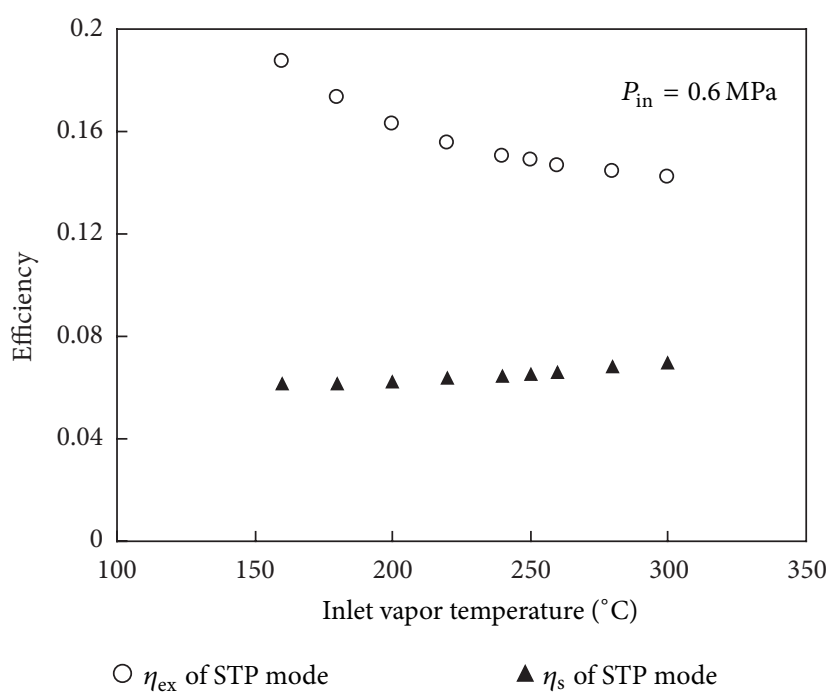

(c)

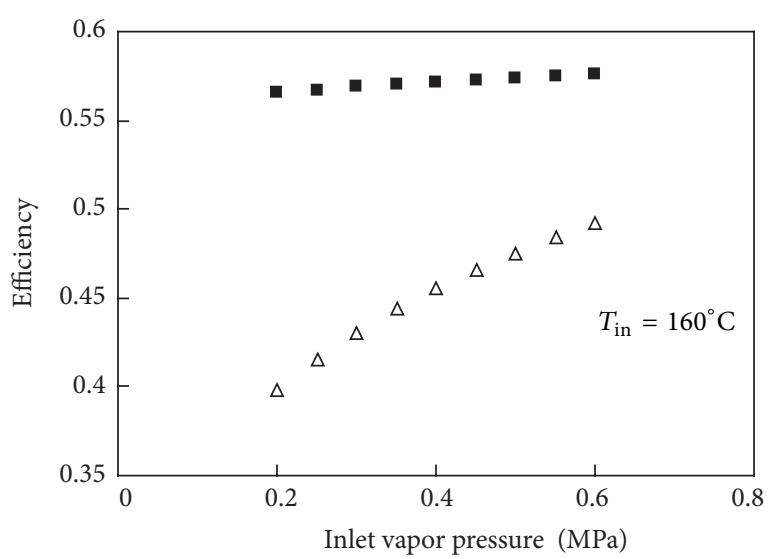

$\triangle \eta_{\text {ex }}$ of CCHP mode

- $\eta_{\mathrm{s}}$ of CCHP mode

(b)

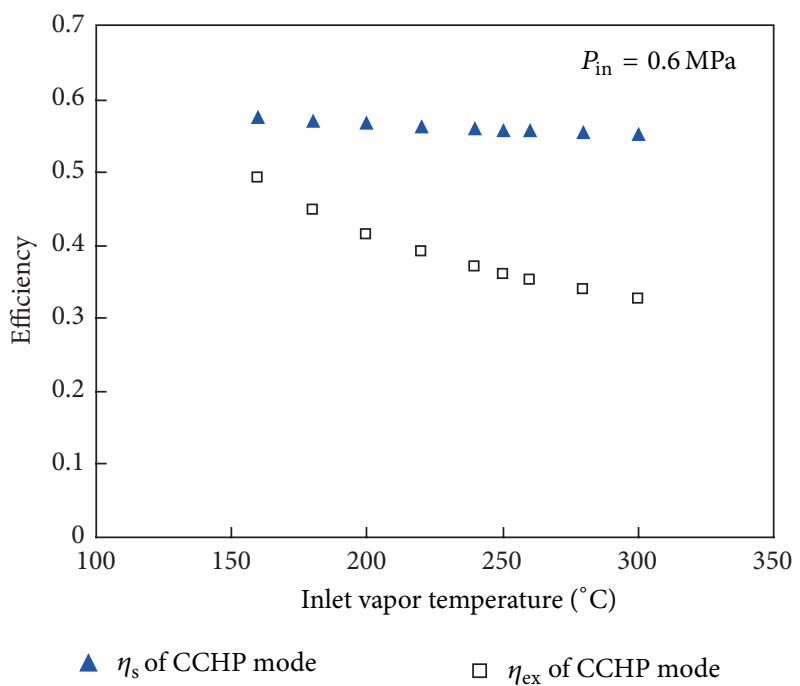

(d)

FIGURE 6: The effects of inlet vapor parameters on the overall efficiencies of energy and exergy.

power systems. It has seven optional operation modes. This makes its operation more flexible. It also can be assembled to be a large system by connecting several modules to meet the requirements of different fields.

Primary calculations show that overall energy and exergy efficiencies of the CCHP operation mode are obviously higher than that of the STP mode when the output electricity is given as a constant. In order to obtain the highest overall performance, a high efficient absorption refrigerator and water heater should be equipped to the modular CCHP system.

Analyses show that the performance of CCHP system is affected by the pressure and temperature of vapor at inlet of the power generator. When the inlet vapor pressure is constant, higher inlet vapor temperature deteriorates both the energy and exergy efficiencies of the CCHP system. On the other hand, higher inlet vapor pressure can improve both the energy and exergy efficiencies of the CCHP system. Thus, if it is available, the inlet vapor pressure should be as high as possible. In this work, the inlet vapor temperature and pressure are mainly determined on the basis of the cost of solar collectors, the available screw expander, and its characteristics when the output electricity is given as $10 \mathrm{~kW}$. Thus, the inlet vapor temperature and pressure of the vapor entering the screw expander are given as $160^{\circ} \mathrm{C}$ and $0.6 \mathrm{MPa}$, respectively. Though the lower inlet vapor pressure may cause lower efficiency of the CCHP system, it can reduce the initial investment.

Anyway, this work makes it possible to efficiently utilize solar thermal power in microdistributed energy systems though only the CCHP and STP operation modes are primarily analyzed and compared. Further research such as optimum design, effect of the solar radiation, characteristics of solar collectors and absorbers, and detailed simulation and experiments will be conducted to investigate the performance characteristics of the CCHP at all operation modes, especially when there are not so many demands for electricity, hot water, or cooling capacity in the next researches. In addition, 
the effect of the thermal storage and the auxiliary heater on the system performance also needs investigation. Fortunately, we are designing an experimental system as a prototype to investigate this CCHP system with the financial support of Shenzhen government. Some theoretical and experimental researches will be carried out soon.

\section{Conflict of Interests}

The author declares that there is no conflict of interests regarding the publication of this paper.

\section{Acknowledgment}

The author is grateful to Shenzhen Science and Technology Innovation Committee for supporting this research (no. JCYJ20130331150226792).

\section{References}

[1] R. L. Pons and T. B. Clark, "A dish-stirling solar-thermal power system," International Journal of Ambient Energy, vol. 1, no. 3, pp. 133-148, 1980.

[2] D. Y. Goswami, "Solar thermal power: status of technologies and opportunities for research," in Proceedings of the 2nd ASMEISHMT Heat and Mass Transfer Conference, pp. 57-60, Tata McGraw-Hill, New Delhi, India, 1995.

[3] Y. You and E. J. Hu, "A medium-temperature solar thermal power system and its efficiency optimisation," Applied Thermal Engineering, vol. 22, no. 4, pp. 357-364, 2002.

[4] S. Lu and D. Y. Goswami, "Optimization of a novel combined power/refrigeration thermodynamic cycle," Journal of Solar Energy Engineering, vol. 125, no. 2, pp. 212-217, 2003.

[5] G. Tamm, D. Y. Goswami, S. Lu, and A. A. Hasan, "Novel combined power and cooling thermodynamic cycle for low temperature heat sources, Part I: theoretical investigation," Journal of Solar Energy Engineering, vol. 125, no. 2, pp. 218-222, 2003.

[6] G. Tamm and D. Y. Goswami, "Novel combined power and cooling thermodynamic cycle for low temperature heat sources, part II: experimental investigation," Journal of Solar Energy Engineering, vol. 125, no. 2, pp. 223-229, 2003.

[7] S. Vijayaraghavan and D. Y. Goswami, "Organic working fluids for a combined power and cooling cycle," Journal of Energy Resources Technology, vol. 127, no. 2, pp. 125-130, 2005.

[8] M. Kane, D. Larrain, D. Favrat, and Y. Allani, "Small hybrid solar power system," Energy, vol. 28, no. 14, pp. 1427-1443, 2003.

[9] F. Xu and D. Y. Goswami, "Thermodynamic properties of ammonia-water mixtures for power-cycle applications," Energy, vol. 24, no. 6, pp. 525-536, 1999.

[10] D. Y. Goswami and F. Xu, "Analysis of a new thermodynamic cycle for combined power and cooling using low and mid temperature solar collectors," Journal of Solar Energy Engineering, vol. 121, no. 2, pp. 91-97, 1999.

[11] F. Xu, D. Yogi Goswami, and S. S. Bhagwat, "A combined power/cooling cycle," Energy, vol. 25, no. 3, pp. 233-246, 2000.

[12] S. Lu and D. Y. Goswami, "Theoretical analysis of ammoniabased combined power/refrigeration cycle at low refrigeration temperatures," in Proceedings of the Solar Engineering 2002, pp.
117-125, American Society of Mechanical Engineers, Reno, Nev, USA, June 2002.

[13] N. Zhang, R. Cai, and N. Lior, "A novel ammonia-water cycle for power and refrigeration cogeneration," in Proceedings of the ASME International Mechanical Engineering Congress and Exposition (IMECE '04), pp. 183-196, ASME, Anaheim, Calif, USA, 2004.

[14] Z. S. Abdel-Rehim, "A new system to produce cooling and power using solar thermal energy," Energy Sources A: Recovery, Utilization and Environmental Effects, vol. 33, no. 17, pp. 16361650, 2011.

[15] A. Mayere and S. Riffat, "A novel solar driven micro-CHP system: a state of the art review," Journal of Energy and Power Engineering, vol. 4, no. 11, pp. 30-35, 2010.

[16] D. Borello, A. Corsini, F. Rispoli, and E. Tortora, "A co-powered biomass and concentrated solar power rankine cycle concept for small size combined heat and power generation," Energies, vol. 6, no. 3, pp. 1478-1496, 2013.

[17] Y. X. Su, Z. D. Fei, and X. X. Yang, "Investigation on the distributed energy system for cooling-heating-power combined cycle driven by solar energy," Energy Engineering, vol. 5, pp. 24$27,2004$.

[18] H. D. Wang, "Experimental study on $\mathrm{LiNO}_{3}-\mathrm{NH}_{3}$ diffusionabsorption refrigeration system," Key Engineering Materials, vol. 474-476, pp. 2335-2340, 2011. 

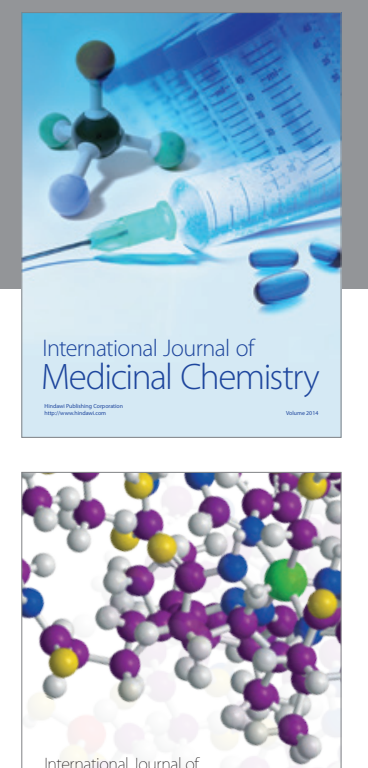

\section{Carbohydrate} Chemistry

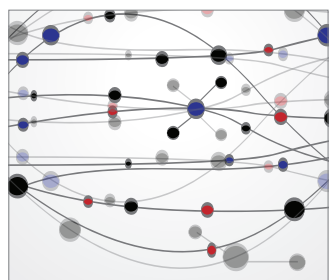

The Scientific World Journal
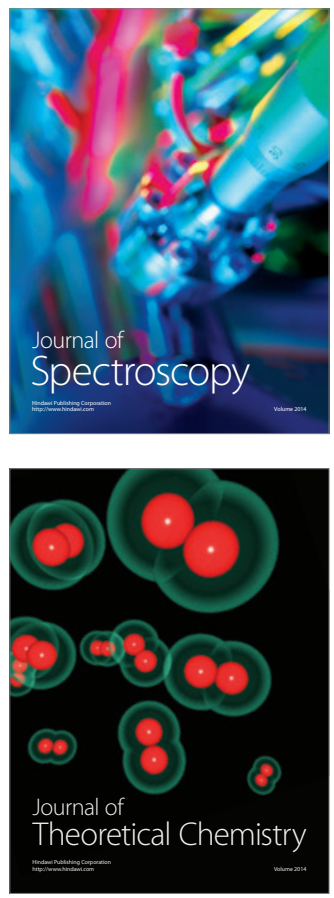
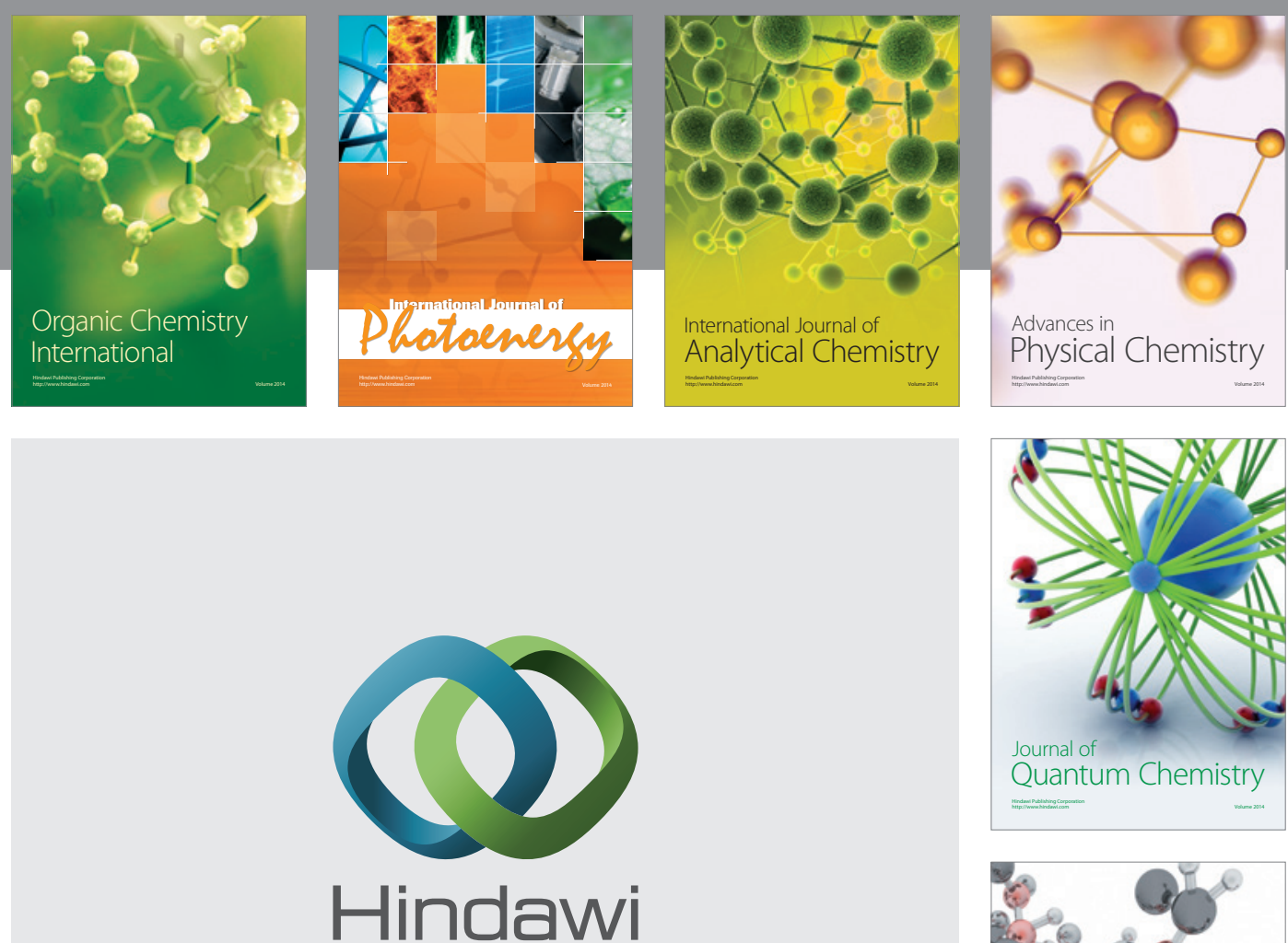

Submit your manuscripts at

http://www.hindawi.com

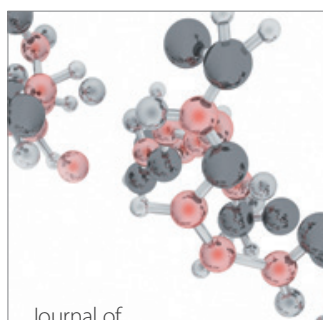

Analytical Methods

in Chemistry

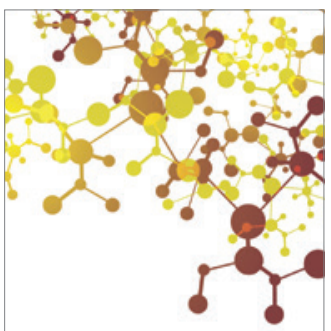

Journal of

Applied Chemistry

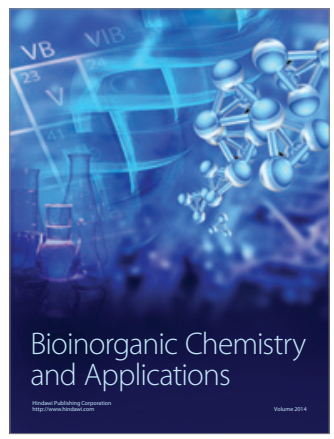

Inorganic Chemistry
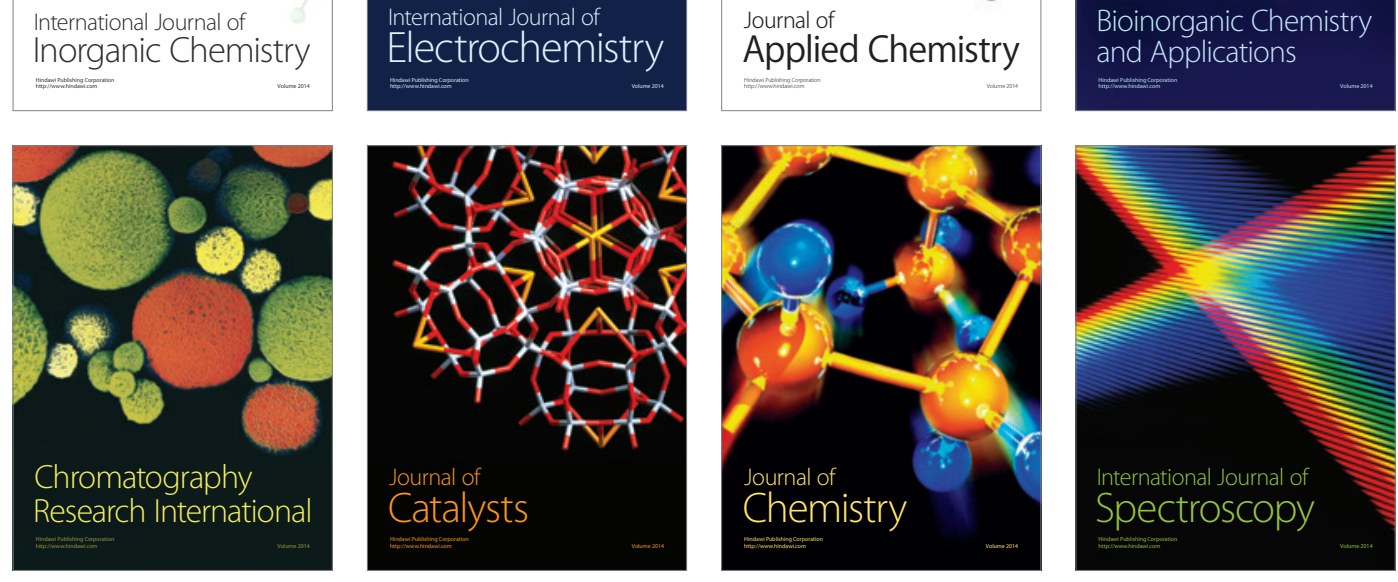